\title{
Formulation, Realization, and Demonstration of a Process to Generate Aerodynamic Metamodels for Hypersonic Cruise Vehicle Design
}

\author{
Robert A. McDonald and Dimitri N. Mavris \\ Aerospace Systems Design Laboratory \\ Georgia Institute of Technology
}

\begin{abstract}
The desire to facilitate the conceptual and preliminary design of hypersonic cruise vehicles has created the need for simple, fast, versatile, and trusted aerodynamic analysis tools. Metamodels representing physics-based engineering codes provide instantaneous access to calibrated tools. Nonlinear transformations extend the capability of metamodels to accurately represent a large design space. Independence, superposition, and scaling properties of the hypersonic engineering method afford an expansive design space without traditional compounding penalties. This one-time investment results in aerodynamic and volumetric metamodels of superior quality and versatility which may be used in many forms throughout early design. As a module, they can be an integral component within a multidisciplinary analysis and optimization package. Aerodynamic polars they produce may provide performance information for mission analysis. Spreadsheet implementations can provide pocket-calculator-like access to explore the design space. Plotting their essence as profiles exposes design trends may be used in rapid tradeoff studies. This work postulates the procedure, details the execution, and exposes the results of generating uncompromised aerodynamic metamodels for hypersonic cruise vehicle design.
\end{abstract}

\section{INTRODUCTION}

There has long been much interest in hypersonic cruse vehicles within the aerospace community. The ability to travel at speeds many times greater than competing vehicles would afford a hypersonic aircraft significant advantages including enhanced survivability against enemy air defenses, reaching time critical targets, rapid global response, and reduction of the cost of access to space. Reusable launch vehicles utilizing hypersonic cruise trajectories could enable access to space in a manner similar to commercial air travel. Strategic bombers and transports would benefit from the speed and survivability of hypersonic flight. Hypersonic standoff and cruise missiles could reach targets at long ranges with shorter flight times without endangering a human operator. A hypersonic strike fighter would capitalize on its ability to penetrate enemy air defenses to strike time critical targets. The advantages a hypersonic vehicle would enjoy provide exciting opportunities for the aerospace community.

Unfortunately, an inability to develop an affordable hypersonic solution has hindered such developments. The design of a hypersonic cruise vehicle presents a myriad of formidable challenges which include high aerodynamic drag and heating, unconventional propulsion systems, a wide range of off-design operating conditions, and the tight integration of airframe, propulsion, and structure. These difficulties are compounded by the lack of existing baseline vehicles, historical databases, and hypersonic experience within the design community. Consequently, designers must rely on physics based analysis methods that facilitate intelligent decisions and compromises. Furthermore, the preliminary designer does not have the luxury of becoming an expert in every discipline involved with vehicle design. Therefore, what is needed is a means of distilling the essence of complex analysis tools into a form that can quickly and easily provide all required information. The reduction in design cycle time resulting from the use of appropriate preliminary design tools would reduce cost, increase design confidence, and allow the consideration of more designs.

Traditional hypersonic aerodynamic analysis tools are not yet practical for early design. Preliminary design tools need to be quick, simple, and versatile. Computational Fluid Dynamics (CFD) tools can provide high fidelity results for diverse problems, but they are slow, expensive, and require expert attention. While the calibrated results of fast engineering codes are well trusted, they can also be awkward, difficult, and overly specialized in use.

Metamodels represent one solution to this problem. They can be developed to embody the trusted results of an engineering code in a simple, clean, and instantaneous 
package. An initial investment in a metamodel may satisfy a variety of design needs for years; thereby becoming the new design database.

Metamodels typically contain compromises between their accuracy and range of applicability. Properly implemented system simplifications and nonlinear transformations allow the creation of metamodels without compromising validity or scope.

\section{FORMULATION}

METAMODELS Metamodels provide an approximation to another physical model, where a set of independent input variables lead to a dependent output variable in a function-like manner. Many approximation techniques may be used as metamodels. These include response surfaces, lookup and interpolation tables, kriging $[1,2]$, neural networks $[3,4]$, and Gaussian processes[5]. While a complete taxonomy of metamodels is beyond the scope of this paper, a discussion of the techniques used in this study is appropriate, refer to Simpson et. al. for a more complete discussion[6]. All the metamodels used herein are based on a finite number of predetermined analyses, analogous to the design of experiments (DOE) or Taguchi methods.

Metamodels may be tailored to provide just the metrics relevant to the designer. In this study, metamodels were devised to capture the effect of aircraft geometry and flight condition upon volume contained and aerodynamic performance.

Once generated, metamodels may be repeatedly incarnated in any of a variety of forms suiting the designer's needs. If insight into design trends is desired, the metamodels may be used to generate charts depicting the influence of the design variables on the chosen metrics. If an interactive design calculator is required, the metamodels may be implemented in a spreadsheet providing instant access to qualitative design trades. When vehicle performance and mission analysis capabilities are desired, metamodels can provide the volume available for fuel and aerodynamic polars. Metamodels may be linked directly to multidisciplinary analysis and design tools, combining their expertise with other fields.

Response Surface Equations A response surface equation (RSE) is a polynomial regression to a data set[7]. The coefficients are chosen such that the surface best matches the data set in a least squares sense. This choice does not guarantee that the original dependant variable value can be recovered from the metamodel. While not generally so limited, the RSEs used in this study were second-order, thereby capturing first-order and second-order influences, as well as all two-way interactions of the independent design variables on the response. The metamodels produced by RSEs are continuous and have continuous derivatives. Once estimated, the only information required to use an RSE as a metamodel are the polynomial coefficients. The general polynomial form of a quadratic RSE is included as Equation 1, where ' $Y$ ' is the dependent response, ' $x$ ' represents the independent design variables, and ' $b$ ' represents the polynomial coefficients.

$$
Y=b_{0}+\sum_{i=1}^{n} b_{i} x_{i}+\sum_{i=1}^{n} b_{i i} x_{i}^{2}+\sum_{i=1}^{n-1} \sum_{j=i+1}^{n} b_{i j} x_{i} x_{j}
$$

High-Order Curve Fits Least squares fits may also be applied to equations containing more terms than a second-order RSE. The additional terms may capture higher-order effects and/or more complex interactions. The high-order curve fits used for this study captured all single variable effects up to fifth-order, and all n-way variable interactions. High-order fits have all the advantages and disadvantages of RSEs, but hold the potential to improve a model at the macroscopic level. This potential comes at the risk of introducing anomalous high-order ripples to the solution, thereby degrading the model at the microscopic level.

Interpolation Interpolation involves estimating the value of a function at a point based on function values known at surrounding points. Higher-order interpolation bases the function estimate on a larger footprint of points surrounding the desired point. In order to use interpolation, a database of points in the design space is required. This presents a larger computational overhead than with a polynomial metamodel approach. Although interpolation techniques are guaranteed to recover the original dependent variable values at the design points. They produce continuous metamodels with discontinuous derivatives. Interpolation inherently captures the effects of all independent variable interactions on the dependent function.

TRANSFORMATIONS Metamodels will typically perform poorly in situations where the mathematical behavior of the actual relationship is significantly different from the behavior of the metamodel being applied. Transformations may be applied to the independent and/or dependent variables in these cases in effort to improve the metamodel. Consult Chapter 8 of the text by Box and Draper [7] for a comprehensive discussion of transformations in modeling.

Any operation performed on a variable involved within a metamodel may be considered a transformation. Linear transformations do not affect the behavior of a metamodel; they only have the effect of uniformly scaling or shifting the scale of a variable. For example, when considering a polynomial regression, transformation of the independent variable ' $X$ ' into ' $a X$ ' or ' $X+a$ ' will only change the polynomial's coefficients, not the prediction capability of the metamodel. Nonlinear transformations alter the behavior of a metamodel by stretching and contracting the 
functional relationship. As a further example, transformation of ' $X$ ' into ' $1 / X$ ' will change the shape of the relationship, thereby altering the prediction capability of the metamodel. Monotonic nonlinear transformations of a positive variable have the effect of contracting the scale of the variable in part of its range, while expanding the scale in the remainder of the range. Power transformations are a subset of this class which commonly have utility in engineering problems. Most of the transformations used herein were power transformations. Because these transformations are pertormed on necessarily positive quantities, it is important that the variable be measured relative to a natural origin. This is often the case in engineering problems where, for example, temperature would be measured on a Kelvin, rather than Celsius, scale.

Dependent variable transformations may be regarded as the uniform application of an alternate transformation to all independent variables. Independent variable transformations provide the freedom to apply a different transformation to each independent variable. Superior control, and therefore impact and accuracy, may be attained through the use of properly. chosen independent variable transformations rather than a solitary dependent variable transformation.

Recognizing that the actual functional form of a quantity being approximated by a metamodel is seldom known, a technique for developing transformations is required. This technique may be decomposed into three steps. First, the need for a transformation must be identified. Second, the variables to be transformed must be isolated. Finally, the specific transformations must be determined.

Situations where transformations are required can often be identified by inspection of the metamodel's results. Patterns apparent in a plot of the prediction error vs. prediction for a metamodel indicate systematic error, which in turn implies the need for a transformation to alter the behavior of the metamodel.

Variables which will be highly sensitive to nonlinear transformations can quickly be identified by inspection of the ratio of maximum to minimum value, ' $X_{\max } / X_{\min }$ ', of the variable being considered. This technique may be applied to dependent and independent transformations alike. If this ratio is small, monotonic nonlinear transformations will be nearly linear in the range of variables being investigated, and will therefore have little impact. If this ratio is large, the dependent variable is a good candidate for a transformation. The indicator, ' $X_{\max } / X_{\min }$ ', provides no information regarding the actual relationship being modeled. This indicator only identifies situations where transformations could potentially have significant impact.

Appropriate transformations may be identified through a number of techniques including dimensional analysis, simplified derivation, empirical suggestions, and various statistical methods. The method of maximum likelihood is a statistical method that may be used to identify depen- dent variable transformations. Iterative linear regression or nonlinear regression techniques may be used to identify the best combination of independent variable transformations. The transformations used in this study were based on the derivation of representative formulas using simple theories.

ANALYSIS TOOLS The Mark $V$ version of the Supersonic/Hypersonic Arbitrary Body Program (S/HABP)[8] was selected as the engineering analysis code from which the metamodels were made. S/HABP is descended from the original Gentry program[9], and has been under development by various contractors for the United States Air Force and NASA for many years. It has become the industry standard hypersonic aerodynamic preliminary design tool. Its validity and engineering utility have been demonstrated and well documented $[10,11,12,13]$.

Rather than calculating and monitoring the reference areas needed for aerodynamic coefficients for every component studied, aerodynamic performance was tracked through the normal and axial force areas ' $N / q$ ' and ' $A / q$ ', where ' $N$ ' and ' $A$ ' represent the normal and axial aerodynamic forces, and ' $q$ ' is the dynamic pressure. Alternatively, these quantities may be interpreted as normal and axial force coefficients calculated with a unit reference area. Because the area dependency was not removed from the aerodynamic metrics, there were instances where the area variation would tend to dominate the aerodynamic metrics. This dominance was largely accounted for through appropriate choices of dependent variable transformations.

S/HABP allows the user to choose from various impact methods to estimate the aerodynamic performance of arbitrary shapes. Following guidelines for the selection of analysis methods within S/HABP established by Moore and Williams[14], the tangent cone analysis technique was applied to all fuselages, and the tangent wedge analysis technique was applied to all wing type bodies.

The surfaces of the bodies required for the impact methods are represented in three dimensions by quadrilateral panels. The geometry files required for S/HABP were generated by the parametric geometry package, Rapid Aircraft Modeler (RAM)[15]. RAM is under development by the Systems Analysis Branch at NASA-Ames, and is capable of converting a simple and parametric definition of an aircraft into a 3-D model, and of calculating its volume.

HYPERSONIC RAMIFICATIONS The aerodynamic impact methods employed by S/HABP involve subjecting each panel to the freestream flow, calculating the resulting pressure and forces, and summing the result for every panel. Consequently, there are no interactions between individual panels, or groups of panels. The aerodynamic forces on a wing are independent of the existence, shape, or position of a fuselage or other body. While this property is not physically intuitive, it is correct in the limit of the hy- 
personic approximations made in S/HABP. Similarly, and quite obviously, the volume contained within a body is independent of any surrounding bodies. Further, the combined volume of a group of bodies is simply the sum of the volumes of the members of the group.

These properties of independence and superposition allow the independent aerodynamic and volumetric investigation of aircraft components. Consequently, a design space exploration may investigate a wide variety of wings and fuselages without concerning itself with the multiplicative combinations of wings and fuselages.

The simple square and cube scaling rules that respectively apply to aerodynamic and volumetric quantities imply that aerodynamic and geometric investigations may be performed on bodies of a uniform size, and the results may be applied to bodies of arbitrary size. Consequently, a design space exploration may investigate a wide variety of bodies without concerning itself with the dimensional size of the bodies. Individually, these simple properties would only facilitate the scaling of a complete aircraft, i.e. if the presence of a fuselage influences the aerodynamic properties of a wing, then the size of the fuselage would obviously influence the aerodynamic properties of the wing. However, when combined with the previously discussed independence and superposition properties, these scaling properties become truly powerful in their ability to simplify the design space.

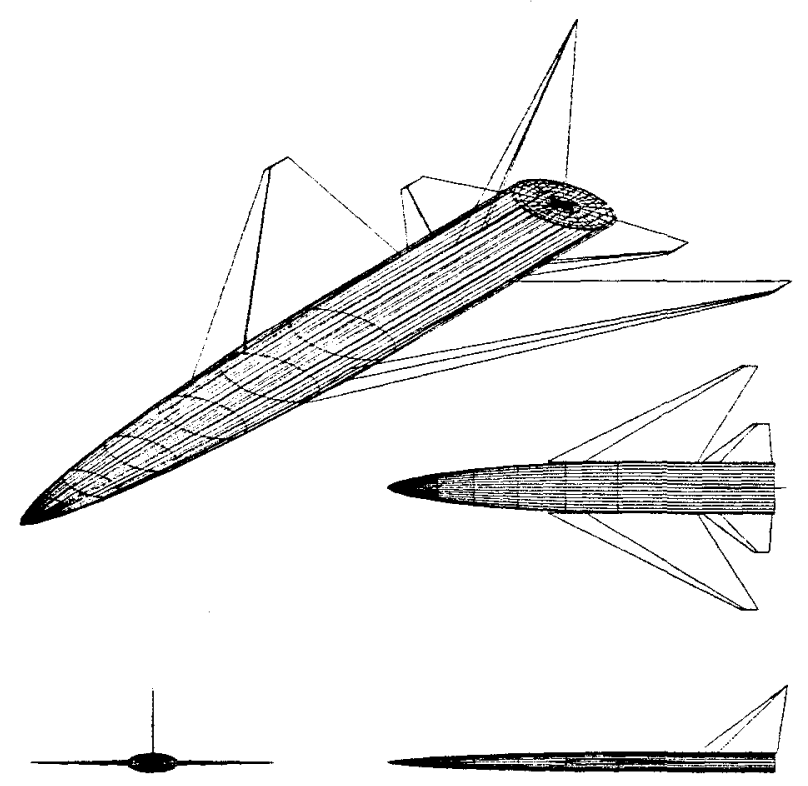

Figure 1: Complete aircraft configuration used to test scaling, independence, and superposition properties of the analyses.

A representative aircraft was analyzed to demonstrate the scaling, independence, and superposition properties. A complete aircraft configuration, depicted in Figure 1, was generated in RAM and analyzed utilizing S/HABP. Components geometrically similar to those used to construct the aircraft were analyzed individually. The results were appropriately scaled, and summed according to the established rules. The built-up volume was exactly equal to the volume of the complete configuration. The resulting drag polars were plotted in comparison as Figure 2. As expected, the aerodynamic behavior of the built-up aircraft was coincident with that of the complete configuration.

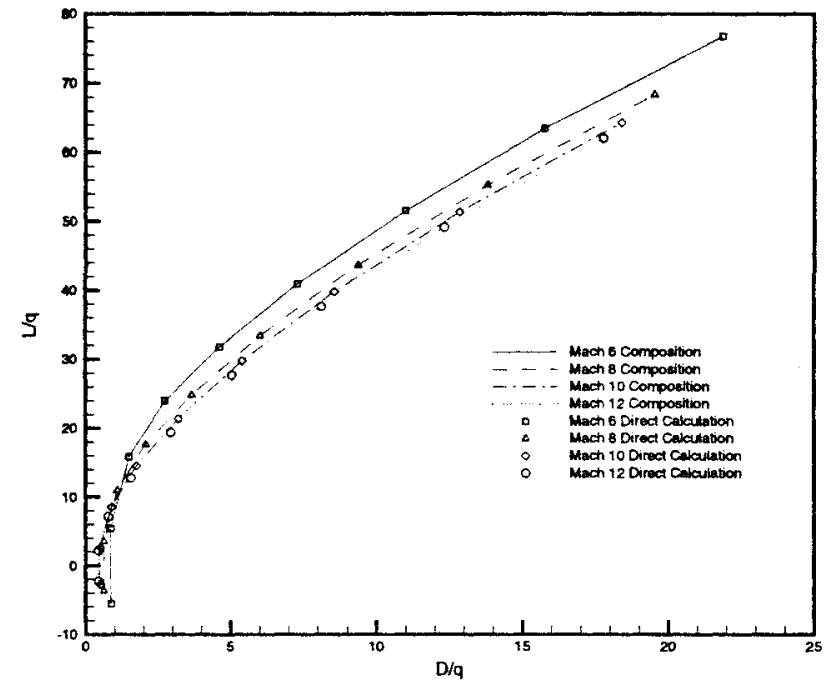

Figure 2: Drag polar for the complete aircraft configuration, comparing direct aerodynamic computation with the superposition of scaled aircraft components.

\section{REALIZATION}

DESIGN SPACE The ramifications of the hypersonic properties discussed above afford a very large design space without incurring the traditional compounding penalty. To facilitate the use of complex metamodels, the predetermined analyses (DOEs) were an ordered, fully populated, and high resolution sample of the design space.

The fuselages investigated were elliptical cylinders with sharp noses and blunt bases. Four parameters defined the fuselage geometry, the width and height of the cylinder and the length and shape (rho) of the nose. Rho is a shape factor defined in RAM, where tho equal to zero results in a blunt nose, tho between zero and one results in a shape similar to a tangent ogive nose, tho equal to one results in a conical nose, and rho greater than one result in shapes similar to a trumpet bell. The ranges of geometric variables included in the fuselage design space were included as Table 1. Planform views of fuselages representing the design space were depicted as Figure 3.

The wings and vertical tails investigated were swept, trapezoidal wings. A diamond airfoil was used, with the maximum thickness occurring at the $30 \%$ chord location. A limitation inherent to RAM prevented changing the maximum thickness location. The parametric variables and DOE used for the wing and vertical tail were identical. Four parameters defined the wing-type geometries. The 
Table 1: Fuselage geometry DOE.

\begin{tabular}{|l|c|c|c|}
\hline Parameter & \# of Points & Min & Max \\
\hline Fuselage Length & 1 & \multicolumn{2}{|c|}{1.00} \\
\hline Height & 10 & 0.05 & 0.30 \\
\hline Width & 10 & 0.05 & 0.30 \\
\hline Nose Length & 10 & 0.05 & 0.75 \\
\hline Nose Rho & 10 & 0.30 & 1.30 \\
\hline
\end{tabular}

Figure 3: Possible fuselage configurations captured within the design space.

aspect ratio, taper ratio, thickness to chord ratio, and the tangent of the leading edge sweep angle. Because RAM works directly with the tangent of the sweep angle, it was used in lieu of the sweep angle itself. This produced a bias in the DOE towards wings with large sweep angles, but did not degrade the quality of the metamodels across the entire range of sweep angles. The ranges of geometric variables included in the wing-type design space were included as Table 2. Planform views of wings representing the design space were depicted as Figure 4.

Table 2: Wing-type geometry DOE.

\begin{tabular}{|l|c|c|c|}
\hline Parameter & \# of Points & Min & Max \\
\hline Wing Span & 1 & \multicolumn{2}{|c|}{1.00} \\
\hline Aspect Ratio & 10 & 0.20 & 5.00 \\
\hline Taper Ratio & 10 & 0.00 & 1.00 \\
\hline Thickness Ratio & 10 & 0.01 & 0.06 \\
\hline $\tan$ (Sweep) & 10 & 0.00 & 6.00 \\
\hline
\end{tabular}

All components were investigated at a variety of flight conditions. Because all of the aerodynamic calculations were inviscid, and sideslip and roll were not investigated, angle of attack, ' $\alpha$ ', and Mach number, ' $M$ ', were sufficient to completely define the flight condition. The range of flight conditions included in the operating space were included as Table 3.

MODELING A series of MATLAB programs were developed to perform the interpolation between the points in the DOE required for some of the metamodels. A statistical

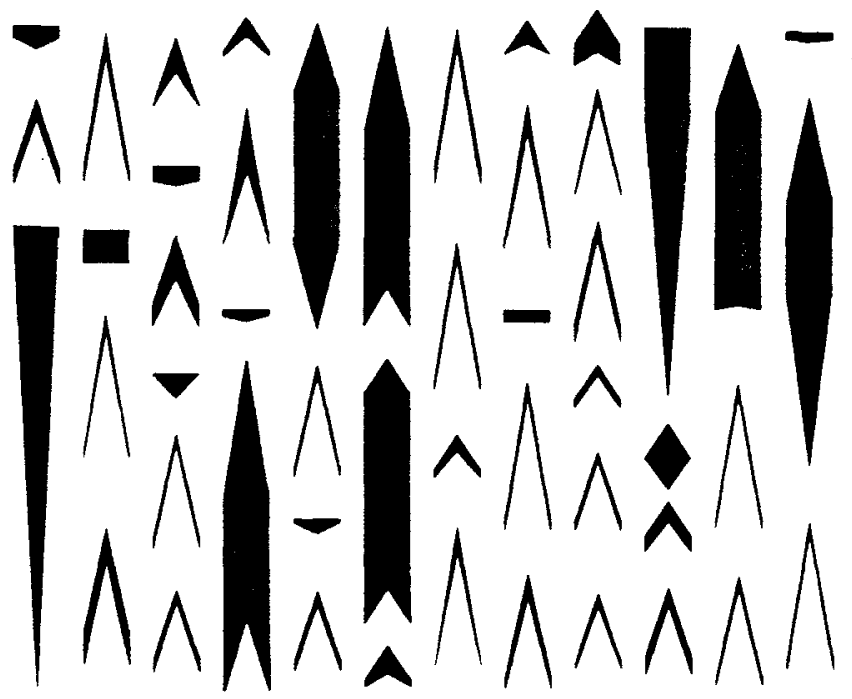

Figure 4: Possible wing configurations captured within the design space.

Table 3: Flight condition DOE.

\begin{tabular}{|l|c|c|c|}
\hline Parameter & \# of Points & Min & Max \\
\hline Mach & 4 & 6.00 & 12.00 \\
\hline Alpha (deg) & 9 & -1.00 & 15.00 \\
\hline
\end{tabular}

package, JMP, was used to perform the least squares regression to the points in the DOE required to estimate the polynomial coefficients for the remainder of the metamodels. The resulting metamodels were exercised over 1,000 cases randomly selected from within the design space. For comparison, the random cases were also analyzed with the traditional suite of programs. Plots depicting the correlation of the actual to predicted values of the random data points were prepared for each of the metamodels. A perfect metamodel will appear as a straight, $45^{\circ}$ line on these plots. The distance a point lies from the perfect $45^{\circ}$ line is indicative of the error in that prediction.

Fuselage As the fuselage geometries are essentially cylinders, their volumetric behavior was rather simple. The only nonlinear influence is that of the nose shape factor, rho. Four metamodels were applied to the fuselage volume. These were linear and cubic interpolation, a second-order RSE, and a high-order fit. The macroscopic performance of the metamodels was depicted in Figure 5. Even though they are of lower-order, the interpolation models were clearly superior to the curve fits. This superiority is a direct result of the inherent ability of interpolation to capture all possible interactions.

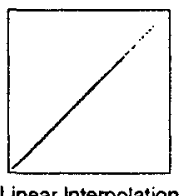

Linear Interpolation

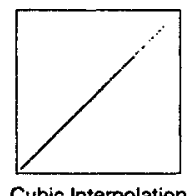

Cubic Interpolation

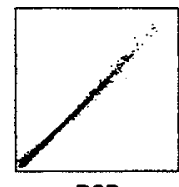

RSE

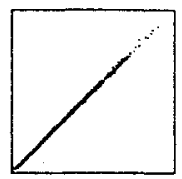

High Order Fit
Figure 5: Correlation of actual to predicted values of fuselage volume. 
As can be observed in Figures 6 and 7, the aerodynamic performance of the fuselage geometries was well modeled by the linear interpolation, but poorly modeled by the curve fits. Because of prohibitive memory requirements, cubic interpolation was not applied as an aerodynamic metamodel for any of the components. AnaIytical application of the Newtonian Flow approximations suggested no geometric transformations. Although the tangent cone technique is based on more complicated shock relations, the Mach number dependence taken from the minimum pressure coefficient correction for Modified Newtonian Flow was implemented to transform ' $M$ ' into ' $1 / M^{2}$ ' for every geometry. With ' $M_{\max } / M_{\min }$ ' of only 2 , it was not surprising that the Mach transformation did not affect any of the metamodels.

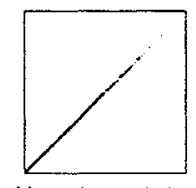

Linear Interpolation

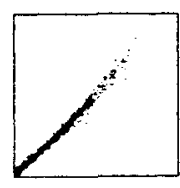

RSE

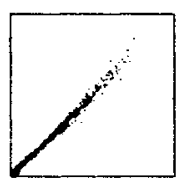

High Order Fit

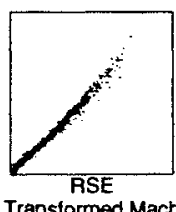

Transformed Mach
Figure 6: Correlation of actual to predicted values of fuselage normal force.

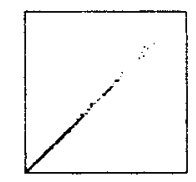

Linear inierpolation

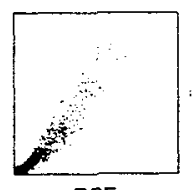

RSE

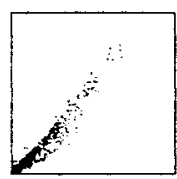

High Order Fit

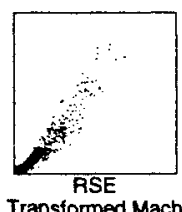

Transformed Mach
Figure 7: Correlation of actual to predicted values of fuselage axial force.

Wing-Type Bodies The volumetric behavior of wing shapes is far more complex than that of cylindrical fuselages. Aspect ratio, ' $A R$ ', was identified as a prime candidate for an independent variable transformation through investigation of ' $A R_{\max } / A R_{\min }$ '. Derivation of a formula for the volume of a plank shaped wing, using the definition of aspect ratio and thickness to chord ratio ' $\tau$ ', revealed that wing volume is directly proportional to ' $T$ ' and inversely proportional to ' $A R^{2}$ '. As can be seen from Figure 8 , the transformation of ' $A R$ ' into ' $1 / A R^{2}$ ' was implemented with much success. Further derivation for the volume ' $V$ ' of a trapezoidal, diamond airfoil wing resulted in Equation 2. Where ' $\lambda$ ' represents the taper ratio, and ' $b$ ' the wing span.

$$
V=\frac{2 \tau b^{3}}{3 A R^{2}}\left(\frac{\lambda^{2}+\lambda+1}{\lambda^{2}+2 \lambda+1}\right)
$$

Not surprisingly, wing volume is independent of sweep angle. The derivation also suggested a complex nonlinear transformation for taper ratio, ' $\lambda_{\max } / \lambda_{\min }$ ' predicted that this transformation could have significant impact. However, the relationship was not sufficiently nonlinear to make a transformation worthwhile. More significant improvement to the metamodel was gained by insuring that complex, n-way, interactions of the independent variables were captured.

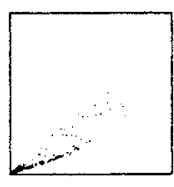

Linear Interpolation

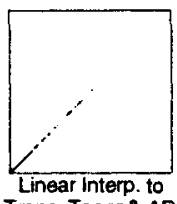

Trans. Taper \& AP

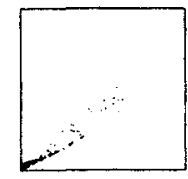

Cubic interpolation
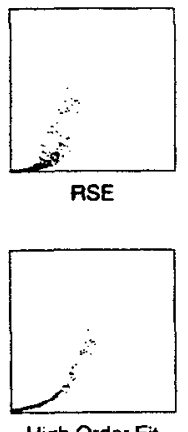

High Order Fit

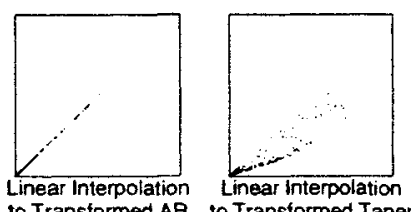

to Transformed AR to Transformed Taper
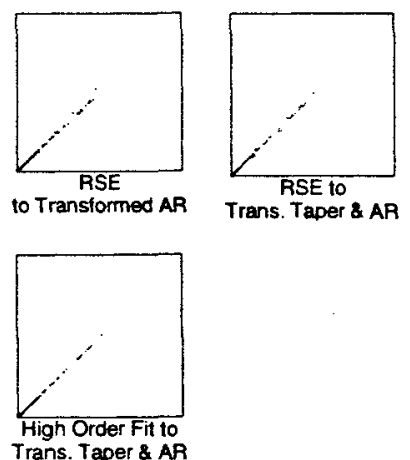

Figure 8: Correlation of actual to predicted values of wing volume.

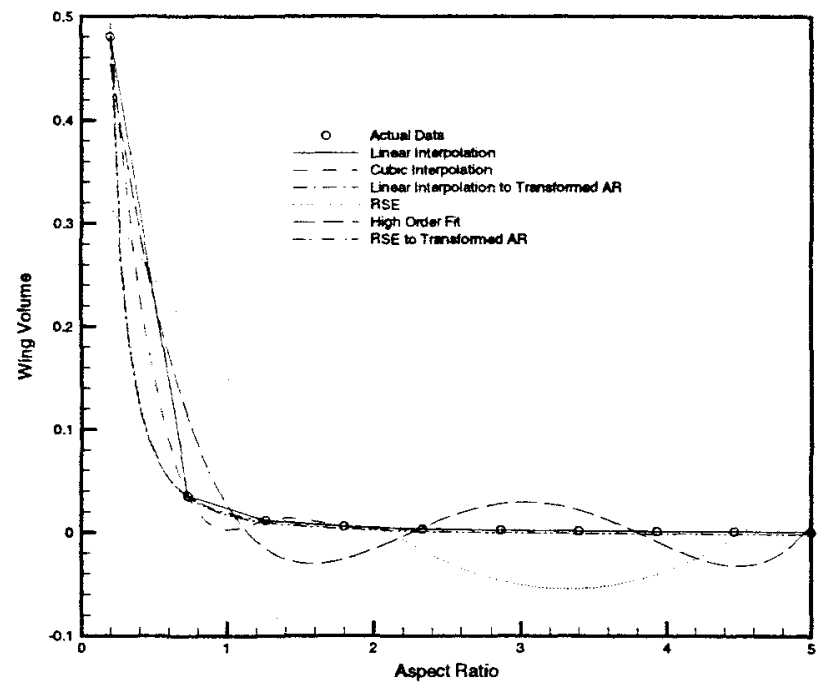

Figure 9: Microscopic behavior of wing volume metamodels.

The importance of appropriate transformations was clearly demonstrated by Figure 9, which depicts the microscopic behavior of a variety of metamodels. Some metamodels whose microscopic behavior was redundant including 'Linear Interpolation to Transformed $A R$ and Taper' and 'RSE to Transformed $A R$ and Taper' were omitted for clarity. Although the quadratic fit of the 'RSE' metamodel did not demonstrate proper trends, the ripples in the 'High-Order Fit' and 'Cubic Interpolation' metamodels emphasize that increasing a model's order for the sake of improving the fit should be avoided. This figure also provides dramatic evidence of the relevance of ' $A R_{\max } / A R_{\min }$ '. If the range of $A R$ was reduced from $0.2-5.0$ to 1.0-5.0, the indicator ratio would have been decreased from 25 to 5 , and a low-order model would have been sufficient without transformation.

The aerodynamic performance metrics for the wing, ' $N / q$ ' 
and ' $A / q$ ', are directly proportional to wing area. Not surprisingly, this influence tends to dominate the metrics. The definition of aspect ratio reveals that wing area is directly proportional to ' $b^{2}$ ' and inversely proportional to ' $A R$ ', thereby suggesting the transformation of ' $A R$ ' into ' $1 / A R$ '. As can be seen from Figures 10 and 11 , this transformation was successful at improving the metamodels. Even though the tangent wedge technique is based on complicated shock relations, the ' $\sin (\theta)^{2}$ ' relationship taken from Newtonian Flow suggested transformations for angular variables. These nonlinear, non-power transformations were implemented for the angle of attack and wing sweep angle, without significant impact.

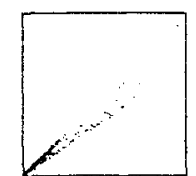

Linear Interpolation

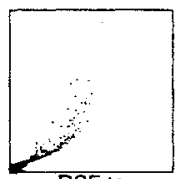

RSE to

Transformed Alpha

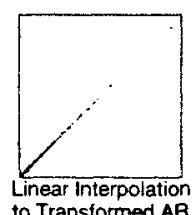
to Transformed AR

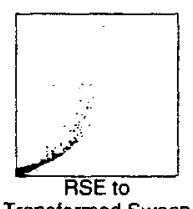

Transformed Sweep

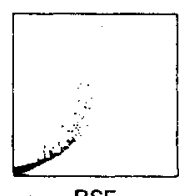

RSE

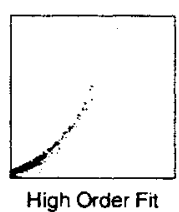

High Order Fit

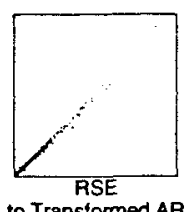

to Transformed AR

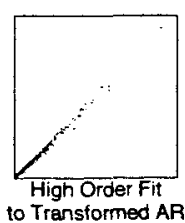

Figure 10: Correlation of actual to predicted values of wing normal force.

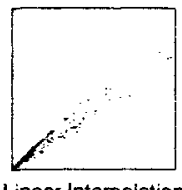

Linear Interpolation

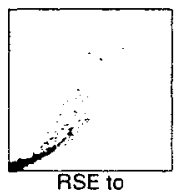

Transtormed Alpha
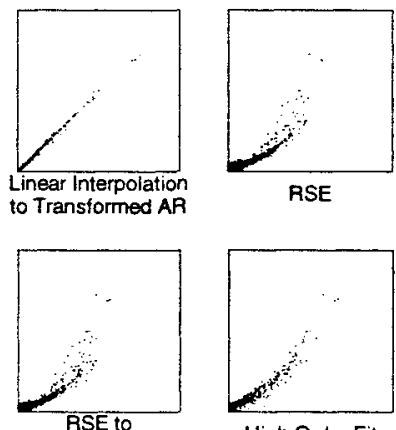

Transtormed Sweep

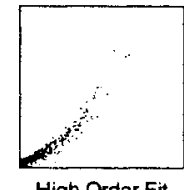

High Order Fit
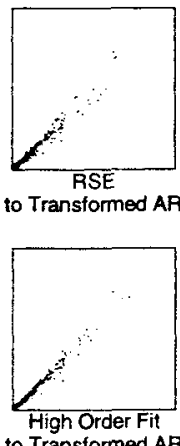

Figure 11: Correlation of actual to predicted values of wing axial force.

Once again, the importance of appropriate transformations was clearly demonstrated by Figure 12, which depicts the microscopic behavior of the wing normal force metamodeis. In a situation where the exact functional relationship between the variables is not known, the transformation reduced the order of the relationship, allowing the least squares fits and other metamodeling techniques to approximate the unknown details of the relationship.

The aerodynamic metrics for the vertical tail are related to the tail area in the same manner as the wing to the wing area. As observed in Figures 13 and 14, the same aspect ratio transformation applied to the wing was successfully applied to the vertical tail.

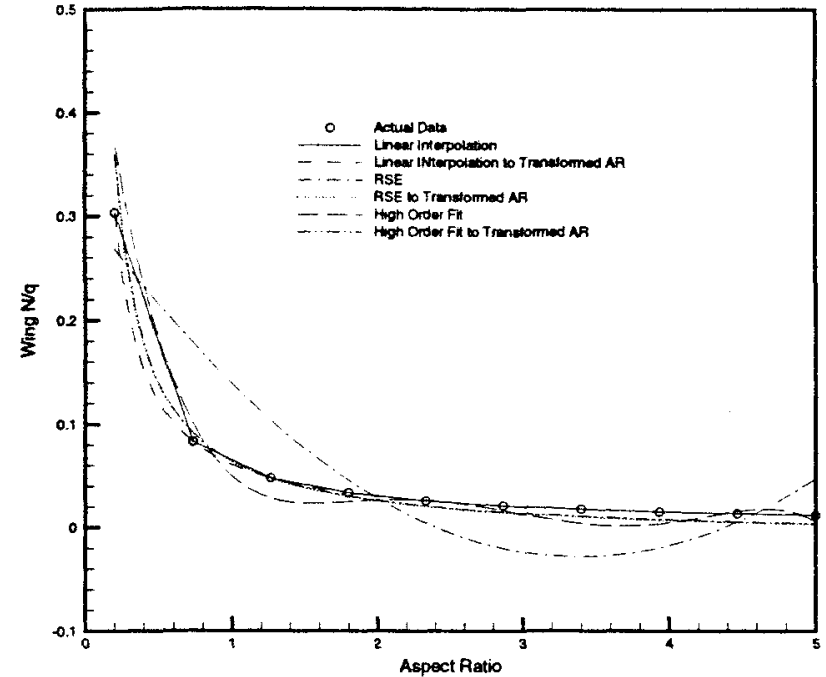

Figure 12: Microscopic behavior of wing normal force metamodels.

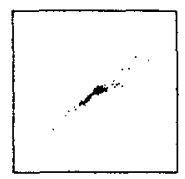

Linear Interpolation
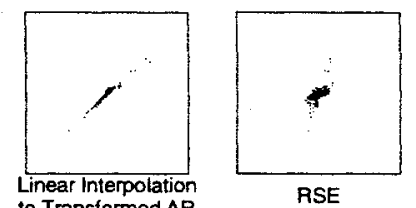

Transolatjon

RSE
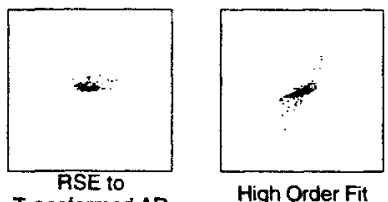

High Order Fit

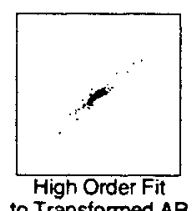

to Transfomed AR

Figure 13: Correlation of actual to predicted values of vertical tail normal force.
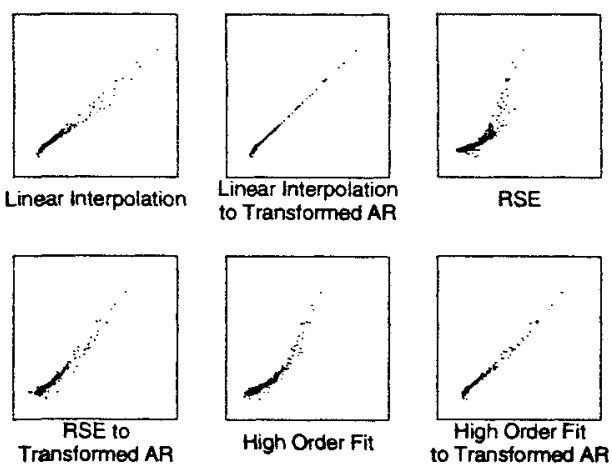

Figure 14: Correlation of actual to predicted values of vertical tail axial force. 


\section{DEMONSTRATION}

Hypersonic aerodynamic metamodels have been developed which are loyal to the analysis codes on both macroscopic and microscopic levels. This investment has the potential of paying a variety of dividends. The metamodels may be used to perform analysis on complete vehicles from a large design space with the same confidence as with the analysis code itself. The function-like nature of the metamodels allows them to be implemented through a variety of interfaces including design charts and tables, spreadsheet applications, or as modules in a multidisciplinary analysis and optimization package. Although the metamodels can not be displayed entirely in this paper, an attempt to convey their essence is worthwhile.

Prediction profiles for all metrics weres included as Figures 15 through 22 . The profiles illustrate the impact of of each of the independent variables on the dependent metric in an isolated, partial derivative sense. On these figures, the solid line depicts each influence to the scale of the largest influence. Whereas the dotted line depicts each influence on an exploded scale. While the uniform scale depicts the relative significance of each variable, the exploded scale conveys an understanding of any trends of lesser magnitude. The dotted line was omitted in cases where the impact of a variable was nonexistant, such as the effect of sweep angle on wing volume in Figure 18, and in cases where the influence of a variable was of the same order as the primary influence, as with the vertical tail normal force metamodel in Figure 21.

Inspection of Figure 15 revealed that the only nonlinear term involved in the fuselage volume was rho, the nose shape factor. Logically, the geometric analogy between fuselage width and height resulted in their having identical impact on the volume contained within the fuselage.
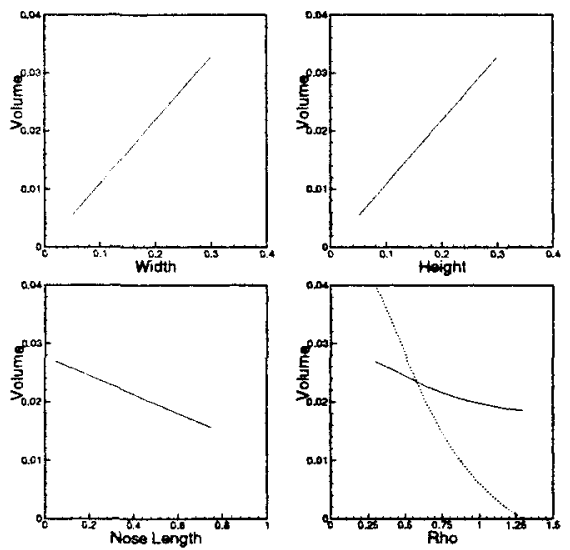

Figure 15: Fuselage volume prediction profiles.

As demonstrated in Figure 16, the width of the fuselage was the most significant influence on the fuselage normal force. A maximum was achieved at low values of both nose length and nose tho. Either of these conditions may result in very blunt noses, which in turn are not modeled well by the tangent cone approximation. This discrepancy makes these particular results suspect, and future investigation should consider employing a different impact method for blunt geometries.
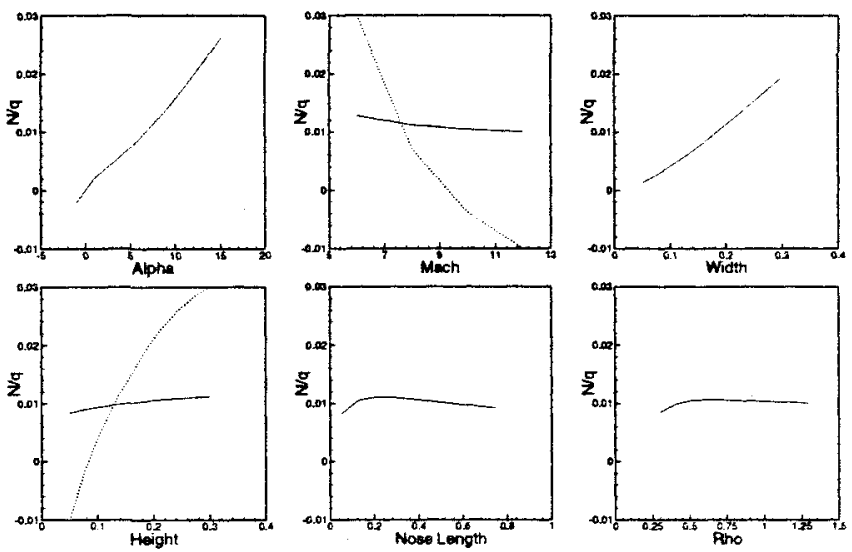

Figure 16: Fuselage normal force prediction profiles.

Figure 17 showed nose length and tho had dramatic impact on the axial force impressed upon the fuselage, overshadowing even the angle of attack. Increasing the nose length reduced axial force monotonically. This is not surprising, considering that long noses correspond directly to high fineness ratios. A minimum axial force was achieved at a nose shape factor of about 0.86 , corresponding directly to a tangent ogive shape.
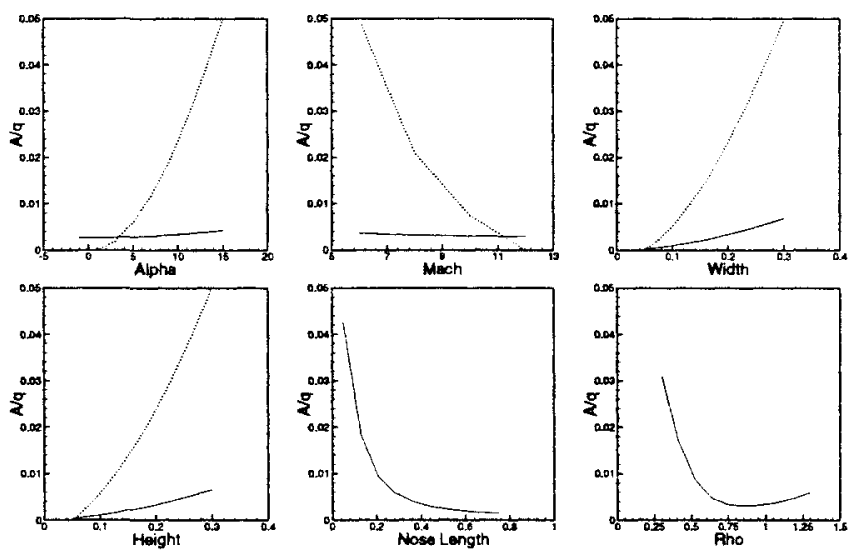

Figure 17: Fuselage axial force prediction profiles.

As predicted by Equation 2, and depicted in Figure 18, wing sweep angle had no impact on the volume contained within the wing-type bodies. Further, the impact of thickness to chord ratio was linear, the taper ratio influence was slightly nonlinear, and aspect ratio was the dominant, and highly nonlinear term.

Interestingly, as revealed by Figure 19, the only significant geometric influence on wing normal force was that of the aspect ratio. This is a direct result of the influence of the aspect ratio on the wing area. The nonlinear transformation inspired by this influence acted to straighten this relationship, thereby aiding the metamodel's ability to capture any remaining influences. Additionally, the taper ratio had no impact on normal force whatsoever. This is a direct 


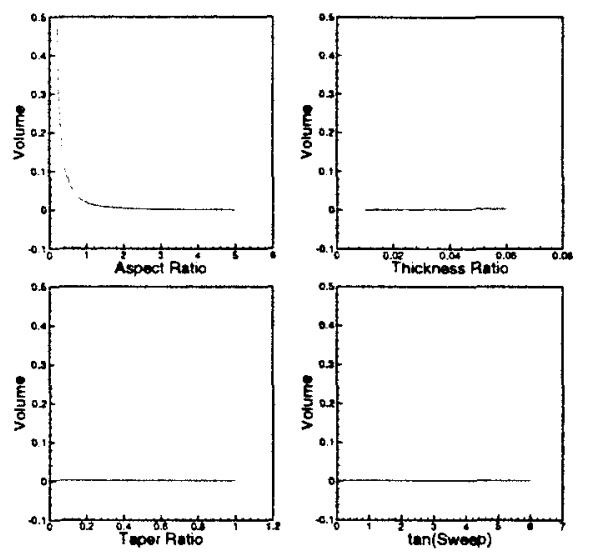

Figure 18: Wing-type volume prediction profiles.

geometric consequence of the fact that taper ratio has no influence on the angle or area of the wing panels; the two determining factors in an impact method. If the wing had been swept along a line other than the leading edge, this taper ratio independence would not have resulted.
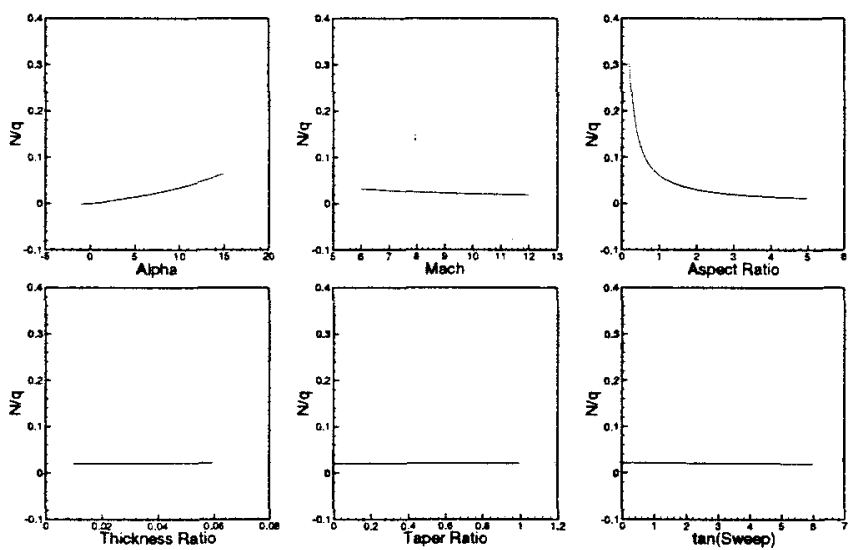

Figure 19: Wing normal force prediction profiles.

Similar to normal force, Figure 20 demonstrated that the wing axial force was totally dominated by the implicit link to wing area within aspect ratio. Intuitively, the second strongest influence was that of the thickness ratio. Wing sweep angle was merely an also-ran, and again the taper ratio had no impact whatsoever.

The vertical tail force influences depicted in Figures 21 and 22 were observed to be quite similar in spirit to the wing force influences. Both normal and axial force were dominated by the area-linked aspect ratio. The only other significant geometric influence was that of the thickness ratio upon axial force. Once more, the taper ratio had no impact on normal or axial force whatsoever.

\section{CONCLUSION}

Opportunities in the aerospace community involving hypersonic cruise vehicles have not been realized because of the significant challenges involved with developing affordable solutions, and the lack of design tools capable
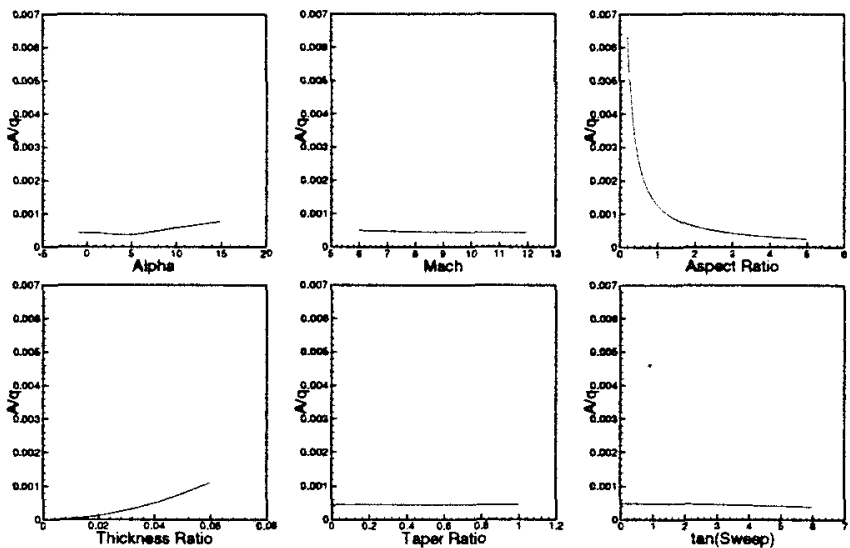

Figure 20: Wing axial force prediction profiles.
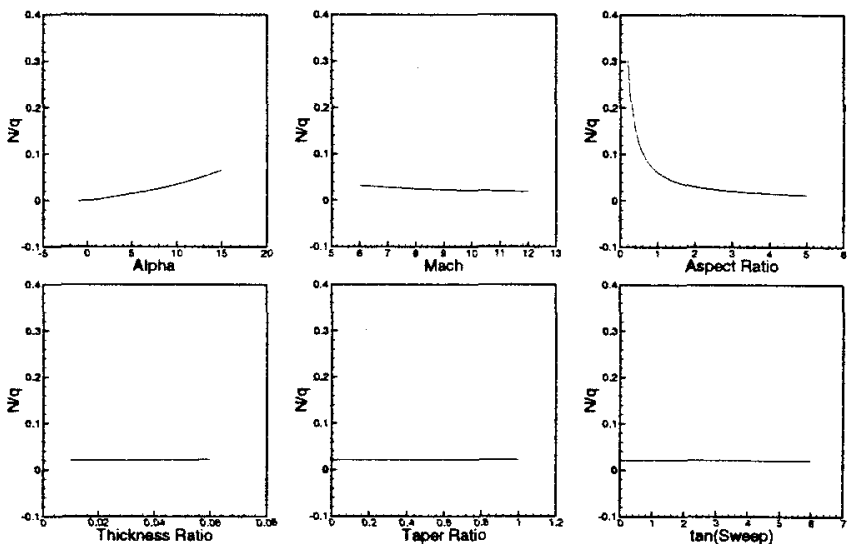

Figure 21: Vertical tail normal force prediction profiles.
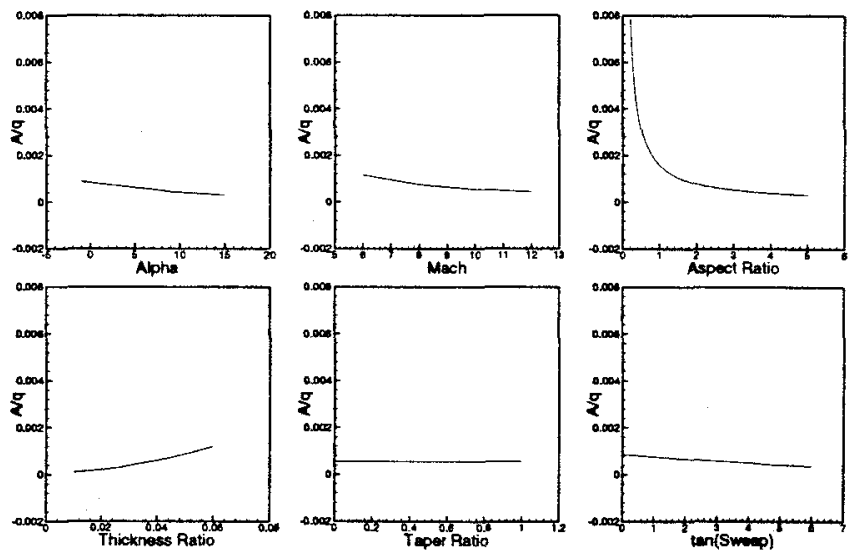

Figure 22: Vertical tail axial force prediction profiles. 
of overcoming such challenges. The metamodels developed herein present a tool with which hypersonic challenges may be attacked. They provide function-like flexibility faithful to industry trusted results.

The techniques presented in this work are not generally without caveats. Without the combined benefits of the superposition, independence, and scaling properties inherent to the hypersonic flow, the compounding penalties in the predetermined analyses would have made the thorough exploration of such a large design space impossible. While the interpolation techniques produce exemplary results, they require fully-populated DOEs. Even though increasing the order of a metamodel can improve its macroscopic behavior, this is done at the risk of sacrificing the microscopic behavior.

Capitalizing on the simplifying properties inherent to the hypersonic analysis code allowed thorough coverage of an enormous design space. Appropriate nonlinear transformations facilitated the accurate modeling of this broad range. Specifically, much success was found by transforming ' $A R$ ' into ' $1 / A R^{2}$ ' and ' $1 / A R$ ' respectively, for metamodels of wing volume and aerodynamic force. A variety of metamodels were explored, each with a set of benefits and drawbacks. Notably, response surfaces provide a simple means of generating smooth models with wide applicability. Interpolation guarantees that all possible interactions will be captured, and that any results present in the DOE will be recovered. The resulting metamodels have the potential of attacking design chalienges at a number of fronts. Among the enabled possibilities are multidisciplinary analysis and optimization, interactive design calculators, mission analysis, design charts, and human understanding and insight.

In addition to opening the world of hypersonic design, the concepts presented in this study leave many opportunities for future work. The design space could be extended through the consideration of more diverse geometries including wave-riders, and wings of varying profile. Automated statistical techniques for identifying appropriate transformations should be tested. Finally, other metamodeling techniques including kriging might be explored to overcome the weaknesses of the current techniques.

\section{REFERENCES}

[1] Noel A. C. Cressie. Statistics for Spatial Data. John Wiley \& Sons, New York, revised edition, 1993.

[2] J. Sacks et. al. Designs and Analysis of Computer Experiments. Statistical Science, vol. 4(no. 4):pp. 409-435, 1989. With discussion.

[3] S. W. Ellacott et. al. Mathematics of Neural Networks: models, algorithms, and applications. Kluver Academic Publishers, Boston, MA, 1997.
[4] T. J. Ross. Fuzzy Logic with Engineering Applications. McGraw-Hill, New York, 1995.

[5] M. A. El-Beltagy et. al.! Metamodeling Techniques for Evolutionary Optimization of Computationally Expensive Problems: Promises and Limitations. In Proceedings of the Genetic and Evolutionary Computation Conference, volume 1, pages 196-203. San Francisco, California: Morgan Kaufmann, 1999.

[6] T. W. Simpson et. al. Metamodels for ComputerBased Engineering Design: Survey and Recommendations. Technical report, Research in Engineering Design, 1997.

[7] George E. P. Box and Norman R. Draper. Empirical Model-Building and Response Surfaces. John Wiley \& Sons, 1987.

[8] Keith A. Burns et. al. Viscous Effects on Complex Configurations. Technical Report WL-TR-95-3060, McDonnell Douglas Aerospace, 1995.

[9] A.E. Gentry et. al. The Mark IV SupersonicHypersonic Arbitrary Body Program. Technical Report AFFDL-TR-73-159, 1973. Volumes I, II, III.

[10] Christopher I. Cruz and Alan W. Wilhite. Prediction of High-Speed Aerodynamic Characteristicsw Using the Aerodynamic Preliminary Analysis System (APAS). AIAA 7th Applied Aerodynamics Conference AlAA 89-2173, American Institute of Aeronautics and Astronautics, July 1989.

[11] Christopher I. Cruz and George M. Ware. Predicted Aerodynamic Characteristics for HL-20 LiftingBody Using Aerodynamic Preliminary Analysis System (APAS). AIAA 17th Aerospace Ground Testing Conference AlAA 92-3941, American Institute of Aeronautics and Astronautics, July 1992.

[12] M. Maughmer et. al. Validation of Engineering Methods for Predicting Hypersonic Vehicle Controls Forces and Moments. Technical Report AIAA 912845, American Institute of Aeronautics and Astronautics, 1991.

[13] Bandu N. Pamadi and Gregory J. Brauckmann. Aerodynamic Characteristics and Development of the Aerodynamic Database of the X-34 Reusable Launch Vehicle. International symposium on atmospheric reentry vehicles and systems, NASA Langley Research Center, March 1999.

[14] M. E. Moore and J. E. Williams. Aerodynamic Prediction Rationale for Analyses of Hypersonic Configurations. 27th Aerospace Sciences Meeting AIAA 890525, American Institute of Aeronautics and Astronautics, January 1989.

[15] J. R. Gloudemans and Paul Davis. Rapid Aircraft Modeler (RAM). Developed by Sterling Software for the NASA-Ames Systems Analysis Branch. 